\title{
PARTICLE SWARM OPTIMIZATION FOR SAGE MAXIMIZATION STEP IN CHANNEL PARAMETER ESTIMATION
}

\author{
Harun Bodur, Celal Alp Tunc, Defne Aktas, Vakur B. Erturk, and Ayhan Altintas \\ Dept. of Electrical and Electronics Engineering, Bilkent University, Ankara, Turkey, e-mail: harun@ee.bilkent.edu.tr
}

Keywords: MIMO; channel estimation; SAGE; particle swarm optimization.

\begin{abstract}
This paper presents an application of Particle Swarm Optimization (PSO) in Space Alternating Generalized Expectation Maximization (SAGE) algorithm. SAGE algorithm is a powerful tool for estimating channel parameters like delay, angles (azimuth and elevation) of arrival and departure, Doppler frequency and polarization. To demonstrate the improvement in processing time by utilizing PSO in SAGE algorithm, the channel parameters are estimated from a synthetic data and the computational expense of SAGE algorithm with PSO is discussed.
\end{abstract}

\section{Introduction}

Recent works have shown that appropriate coding using multiple antennas at the transmitter and receiver can increase the capacity of mobile systems [5]. Such systems are called Multiple Input Multiple Output (MIMO) systems. Design and optimization of MIMO systems require realistic model of the propagation channel. In other words, model needs to characterize the properties of each propagating path such as delay, angles (azimuth and elevation) of arrival (AoA) and departure (AoD), Doppler frequency and polarization [6]. To generate accurate channel models, extensive channel measurements and high resolution estimation tools are required.

Various estimation tools have been used to estimate the channel parameters such as Multiple Signal Classification (MUSIC), Estimation of Signal Parameter via Rotational Invariance Techniques (ESPRIT) and maximumlikelihood (ML) methods like Expectation Maximization (EM) and Space Alternating Generalized Expectation Maximization (SAGE) algorithms [2]. ML methods yield more accurate results and provide higher resolution than other methods, but computational complexity is high due to the brute force search required to find the likelihood maximizing parameters.

The SAGE algorithm is a popular technique for parameter estimation. It updates each parameter sequentially and successively, so it has a lower complexity and a faster convergence [1]. The algorithm has two steps: expecta- tion (E) and maximization (M) steps.

The most computationally intensive part of the SAGE algorithm is in the M-step, and hence, fast search procedures are required to reduce the computational complexity. In this paper, we intend to accelerate the SAGE algorithm using PSO for the search procedure. PSO is a popular evolutionary computation technique which is based on intelligence and movement of swarms [4]. It is shown that PSO is an effective algorithm and has a low computational complexity for solving optimization problems. By using PSO, SAGE algorithm converges rapidly and this convergence will be shown in Section 6.

The signal model for MIMO channels is summarized in Section 2. Section 3 provides a brief introduction to SAGE algorithm. Section 4 explains how PSO can be utilized in SAGE algorithm. Section 5 presents the performance of the proposed algorithm for a synthetic data. We conclude with some remarks in Section 7.

\section{MIMO Signal Model}

In a typical MIMO channel environment shown in Figure 1 there exists a transmitter antenna array $(\mathrm{Tx})$, a receiver antenna array $(\mathrm{Rx})$ and the propagation paths of the transmitted signal. Obstacles that are located in the environment cause reflection, diffraction and refraction. Therefore, the transmitted signal propagates to the receiver through a certain number of paths $(1,2 \ldots L)$. When the transmitted signal travels from $\mathrm{Tx}$ to $\mathrm{Rx}$ on a path, it is delayed, its amplitude is attenuated and its phase is changed. With this knowledge at hand the received signal vector coming from any one of the paths can be written as;

$$
\begin{gathered}
\mathbf{s}\left(t ; \boldsymbol{\Theta}_{l}\right)=\exp \left\{j 2 \pi \nu_{l} t\right\} \mathbf{C}_{R_{x}}\left(\boldsymbol{\Omega}_{R_{x}, l}\right) \mathbf{A}_{l} \\
\mathbf{C}_{T_{x}}\left(\boldsymbol{\Omega}_{T_{x}, l}\right) \mathbf{u}\left(t-\tau_{l}\right)
\end{gathered}
$$

where $\mathbf{u}(\mathrm{t})$ denotes the transmitted signal vector, $\Theta_{l}$ given by

$$
\boldsymbol{\Theta}_{l}=\left[\Omega_{R_{x}, l}, \Omega_{T_{x}, l}, \tau_{l}, \nu_{l}, \mathbf{A}_{l}\right]
$$




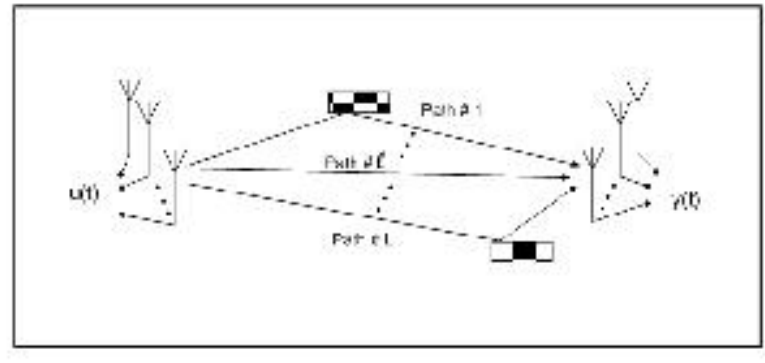

Figure 1, MMO cisannel environment.

is a vector that consists of channel parameters such as angles (azimuth and elevation) of arrival $\left(\Omega_{R_{x}}\right)$, angles (azimuth and elevation) of departure $\left(\Omega_{T_{s}}\right)$, delay $(n)$, Doppler frequency $(n)$ and the polarization matrix $\left(A_{4}\right)$. $\Omega$ is a urit vector that describes the direction from a reference point and given as

$$
\Omega=[\cos (\phi) \sin (\theta), \sin (\phi) \sin (\theta), \cos (\theta)]^{T^{T}},
$$

with $\theta$ and $\phi$ denoting the elevation and azimuth angdes, respectively. Finally in equation (1), $\mathrm{C}_{T_{x, 0}}(\Omega)$ and $\mathrm{C}_{R_{x, \mathrm{Q}}}(\Omega)$, the steering vectors for the transmit and recoive arrays, are given by

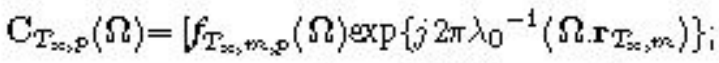

$$
\begin{aligned}
& m=1, \ldots, M]^{T}(p=v, h)
\end{aligned}
$$

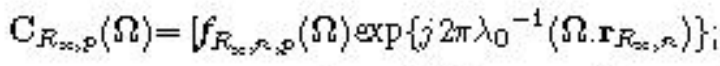

$$
\begin{aligned}
& n=1, \ldots, M^{T}(p=v, h) .
\end{aligned}
$$

where $p$ denotes the polarization type (either vertical (v) or horizontal (h)), $f$ is the field pattern of anternas, $r$ is antenna location, $M$ is number transmitter antennas and $N$ is number receiver antermas. Equation (1) models signal of one path for an antenna and total signal at the receiver is as follows:

$$
\mathbf{Y}(t)=\sum_{l=1}^{L} s\left(t_{i} \Theta_{l}\right)+W(t)
$$

Here $W$ is the additive white Gaussian mise which is assumed to be independent identically distributed Gaussian.

\section{SAGE Algorithm}

SAGE algorithm is an ML estimation method that has been used for different applications like chamel par ameter estimation

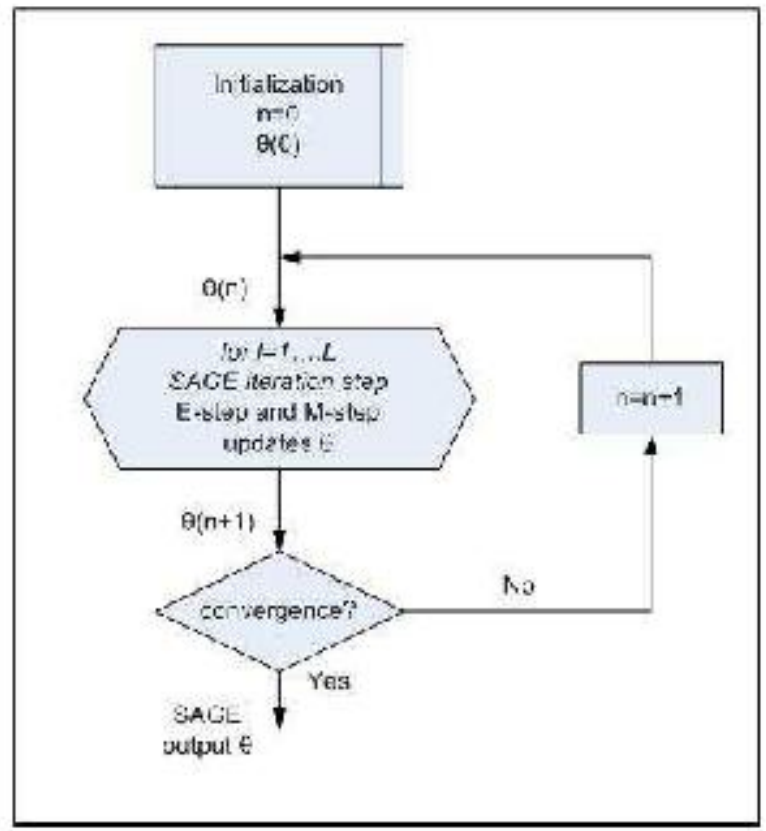

Figure 2, MMO channel environment.

Figure 2 is the flow graph of the SAGE algorithm. The algorithm has two steps, namely, expectation (E) and maximization (M) steps. In an E-step, the expected value of one path at a receiver antenna output is computed. In an M-step, the likelihood function is maximized for parameters of one path sequentially. For each iteration, these steps are performed for all paths and SAGE algorithm stops until all parameters converge.

The formulas of SAGE algorithm depend on two concepts: unobservable admissible data and observable incomplete data. $\mathrm{Y}(t)$ described in equation (6) is the incomplete data and the admissible data for one path is

$$
\mathrm{X}_{4}\left(t^{\prime}\right)=s\left(t_{1} \Theta_{4}\right)+\mathrm{W}\left(t^{\prime}\right)
$$

\section{$3.1 \quad$ E-step}

Since $X_{1}$ is an unobservable function, estimate of this function is based on the observable data vector $\mathrm{Y}(t)$ by

$$
\left.\mathrm{X}_{\imath}(t)=\mathrm{Y}(t)-\sum_{u=1, i \neq l}^{L} \mathrm{~s}_{\left(t_{i}\right.} \Theta_{i l}\right)
$$

where $\Theta l$, is the estimated parameters for $l^{\prime}$ th path from previous iteration(s)[2].

Thus, estimation of admissible data of one path $\left(\mathrm{X}_{1}(t)\right)$ can be found by subtracting estimated signals of all other paths from observed data $\mathbf{Y}(t)$. 


\subsection{M-step}

The computation of parameters is done from the likelihood function and this function is maximized for the parameters individually. The SAGE coordinate-wise updates for the parameters are derived in [6] and given as follows,

$$
\begin{aligned}
& \hat{\tau}_{l}=\arg \max _{\tau_{l}} \mid z\left(\hat{\phi}_{T_{x}, l}, \hat{\theta}_{T_{x}, l}, \hat{\phi}_{R_{x}, l}, \hat{\theta}_{R_{x}, l}, \tau_{l}, \hat{\nu}_{l}\right. \\
& \left.; \hat{x}_{l}\right) \mid \\
& \hat{\nu}_{l}=\arg \max _{\nu_{l}} \mid z\left(\hat{\phi}_{T_{x}, l}, \hat{\theta}_{T_{x}, l}, \hat{\phi}_{R_{x}, l}, \hat{\theta}_{R_{x}, l}, \hat{\tau}_{l}, \nu_{l}\right. \\
& \left.; \hat{x}_{l}\right) \mid \\
& \hat{\phi}_{T_{x}, l}=\arg \max _{\phi_{T_{x}, l}} \mid z\left(\phi_{T_{x}, l}, \hat{\theta}_{T_{x}, l}, \hat{\phi}_{R_{x}, l}, \hat{\theta}_{R_{x}, l}, \hat{\tau}_{l}, \hat{\nu}_{l}\right. \\
& \left.; \hat{x}_{l}\right) \mid \\
& \hat{\theta}_{T_{x}, l}=\arg \max _{\theta_{T_{x}, l}} \mid z\left(\hat{\phi}_{T_{x}, l}, \theta_{T_{x}, l}, \hat{\phi}_{R_{x}, l}, \hat{\theta}_{R_{x}, l}, \hat{\tau}_{l}, \hat{\nu}_{l}\right. \\
& \left.; \hat{x}_{l}\right) \mid \\
& \hat{\phi}_{R_{x}, l}=\arg \max _{\phi_{R_{x}, l}} \mid z\left(\hat{\phi}_{T_{x}, l}, \hat{\theta}_{T_{x}, l}, \phi_{R_{x}, l}, \hat{\theta}_{R_{x}, l}, \hat{\tau}_{l}, \hat{\nu}_{l}\right. \\
& \left.; \hat{x}_{l}\right) \mid \\
& \hat{\theta}_{R_{x}, l}=\arg \max _{\theta_{R_{x}, l}} \mid z\left(\hat{\phi}_{T_{x}, l}, \hat{\theta}_{T_{x}, l}, \hat{\phi}_{R_{x}, l}, \theta_{R_{x}, l}, \hat{\tau}_{l}, \hat{\nu}_{l}\right. \\
& \left.; \hat{x}_{l}\right) \mid \\
& \hat{\mathbf{A}}_{l}=\left(\left|\mathbf{C}_{T_{x}, p}(\hat{\boldsymbol{\Omega}})\right|\left|\mathbf{C}_{R_{x}, p}(\hat{\boldsymbol{\Omega}})\right| P T_{s c}\right)^{-1} \\
& z\left(\hat{\phi}_{T_{x}, l}, \hat{\theta}_{T_{x}, l}, \hat{\phi}_{R_{x}, l}, \hat{\theta}_{R_{x}, l}, \hat{\tau}_{l}, \hat{\nu}_{l} ; \hat{x}_{l}\right)
\end{aligned}
$$

where arg max stands for the argument of the maximum, $P$ denotes signal power, $T_{s c}$ denotes the sensing period of a receiver antenna and $z$ is likelihood function which is given by

$$
z\left(\overline{\boldsymbol{\Theta}}_{l} ; x_{l}\right)=\tilde{\mathbf{C}}_{R_{x}}\left(\boldsymbol{\Omega}_{R_{x}, l}\right)^{H} \mathbf{X}_{l}\left(t ; \tau_{l}, \nu_{l}\right) \tilde{\mathbf{C}}_{T_{x}}\left(\boldsymbol{\Omega}_{T_{x}, l}\right)^{*}
$$

In Equation (16) (.) ${ }^{*}$ denotes the conjugate, $(.)^{H}$ denotes the Hermitian operators and $(\tilde{)})$ denotes normalization.

For the initialization procedure the successive interference cancelation is used and for the details of the derivation reader may refer to [6].

\section{Particle Swarm Algorithm for SAGE}

As we see from the formulas, the algorithm should search for the parameter values maximizing the likelihood function. Since the search domain is continuous this is a tedious operation requiring efficient search procedures. To this end we propose to use PSO to perform the optimization.

PSO is one of the evolutionary computation techniques developed for non-linear optimization problems with continuous valued parameters though it can also be used with discrete variables [4]. The procedure is based on researches on swarms like bees and bird flocking and inspired by social behavior of swarms [4]. This procedure is simple and its computation time is short.

According to the biological research, swarms find their food collectively not individually; information is shared within the members of swarm. In the swarm, each member's position and its information are known, so each member's position and velocity are modified.

Swarm movement optimizes a certain objective function. Each member knows its best value (pbest) and its position. Moreover, each member knows the best value in the swarm (gbest) among pbests. Each member wants to change its position according to its velocity and the distance to pbest and gbest. Velocity and position of each member can be calculated by the following equations:

$$
\begin{gathered}
v=w * v+c 1 * \text { rand } *(\text { pbest }- \text { present }) \\
+c 2 * \text { rand } *(\text { gbest }- \text { present }) .
\end{gathered}
$$

$$
\text { present }=\text { present }+v * t .
$$

Here $v$ is the member velocity, $w$ is a scaling factor, present is the current member's position, rand is a random number between $(0,1) . c 1, c 2$ are learning factors, usually taken as $c 1=c 2=2$.

\section{Performance of SAGE with PSO for a Synthetic Channel Data}

To investigate the performance of SAGE algorithm together with PSO, a synthetic data is created. In the created scenario, double polarized six antennas are used both Tx and Rx. $L=5$ waves are propagating between $\mathrm{Rx}$ and $\mathrm{Tx}$, and the parameters are chosen as in Table 1 . The antenna input signal consists of 55 rectangular pulses with 10 nanoseconds duration, the received signal $(y(t))$ is sampled at $1 \mathrm{GHz}$ and signal to noise ratio (SNR) is taken as $40 \mathrm{~dB}$.

The channel parameters are estimated by SAGE algorithm using three different search procedures: PSO, random search and dogleg. Random Search means sampling the cost function within the reference domain and then 


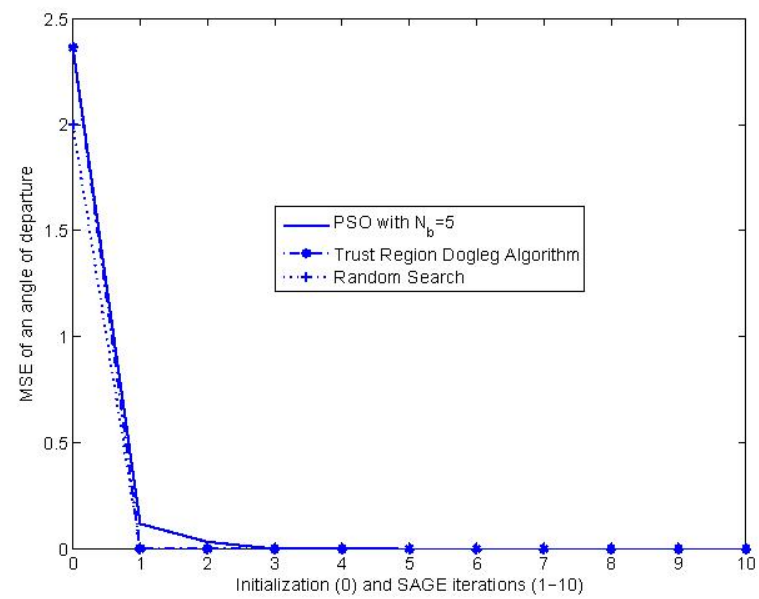

Figure 3. MSE of an angle of departure

\begin{tabular}{|c|c|c|c|c|c|}
\hline $\begin{array}{c}\text { channel } \\
\text { parameters }\end{array}$ & $\ell=1$ & $\ell=2$ & $\ell=3$ & $\ell=4$ & $\ell=5$ \\
\hline $\begin{array}{c}\tau_{l} \\
\text { (nsecs) }\end{array}$ & 25 & 35 & 45 & 55 & 65 \\
\hline $\begin{array}{c}\text { AoA } \\
\text { azimuth }\left(^{\circ}\right)\end{array}$ & 100 & 120 & 140 & 160 & 200 \\
\hline $\begin{array}{c}\text { AoA } \\
\text { elevation }\left(^{\circ}\right)\end{array}$ & 20 & 40 & 60 & 80 & 100 \\
\hline $\begin{array}{c}\text { AoD } \\
\text { azimuth }\left(^{\circ}\right)\end{array}$ & 300 & 330 & 20 & 50 & 70 \\
\hline $\begin{array}{c}\text { AoD } \\
\text { elevation }\left(^{\circ}\right)\end{array}$ & 10 & 30 & 50 & 70 & 80 \\
\hline
\end{tabular}

Table 1. Created synthetic data parameters.

choosing maximum of that function and dogleg is a well known algorithm [3].

We observed that the SAGE algorithm estimated the parameters correctly and rapidly. Figure 3 shows MSE of an angle of departure versus initialization of SAGE algorithm (0) and SAGE iteration numbers (1-10). From Figure 3, SAGE algorithm converges in 3 iterations for all three algorithms. However, computation time for three approaches are significantly different. Table 2 shows the computation time of search procedures both at initialization and SAGE iterations. Table 2 illustrates that PSO's computation time for each iteration is significantly better than other methods.

\section{Conclusion}

In this paper we presented an application of particle swarm optimization in SAGE algorithm for channel parameter estimation. PSO provides an efficient way to perform optimization of the likelihood function for the maximization step of the SAGE algorithm.

To demonstrate the gains in computational complexity by utilizing PSO in SAGE algorithm, we estimated the channel parameters from a synthetically generated data. We observed that although there is no gain in convergence

\begin{tabular}{|c|c|c|}
\hline $\begin{array}{c}\text { Estimation of } \\
\text { one path with }\end{array}$ & $\begin{array}{c}\text { Computation } \\
\text { time of one } \\
\text { iteration } \\
\text { (seconds) }\end{array}$ \\
\hline Random Search & Initialization & 120 \\
& Sage iteration & 1.67 \\
\hline Dogleg & Initialization & 1.9 \\
\hline PSO & Sage iteration & 1.5 \\
\hline & Initialization & 1.75 \\
Sage iteration & 0.025 \\
\hline
\end{tabular}

Table 2. Computational expense of the algorithm compared with other methods. The algorithm works in Matlab environment on an AMD Athlon 3800 processor.

rate in terms of number of iterations, the computation time for each iteration is significantly improved by PSO compared to other search procedures. Therefore PSO can also be applied to channel parameter estimation problem to provide an efficient optimization.

\section{References}

[1] J. A. Fessler and A. O. Hero, "Space-alternating generalized expectation maximization algorithm," IEEE Trans. on Signal Processing, vol. 42, no. 10, pp. 26642677 , Oct. 1994.

[2] B. H. Fleury, D. Dahlhaus, R. Heddergott, and M. Tschudin, "Wideband angle of arrival estimation using the SAGE algorithm," in Proc. IEEE Fourth Int. Symp. Spread Spectrum Techniques and Applications (ISSSTA '96), pp. 79-85, Mainz, Germany, Sept. 1996.

[3] M.J.D. Powell, "A Fortran subroutine for solving systems of nonlinear algebraic equations," Numerical Methods for Nonlinear Algebraic Equations, (P. Rabinowitz, ed.), Ch. 7, 1970.

[4] J. Robinson and Y. Rahmat-Samii, "Particle swarm optimization in electromagnetics," IEEE Trans. Antennas Propag., vol. 52, no. 2, pp. 397-407, Feb. 2004.

[5] I. E, Telatar. "Capacity of multi-antenna gaussian channels," European Transactions on Telecommunication, vol. 10, pp. 585595, Nov/Dec. 1999.

[6] X. Yin, B. H. Fleury, P. Jourdan, and A. Stucki, "Polarization estimation of individual propagation paths using the SAGE algorithm," in Proceedings of the IEEE International Symposium on Personal, Indoor and Mobile Radio Communications (PIMRC), Beijing, China, Sept. 2003. 